\title{
EFFECTS OF ELEMENT DISTORTIONS ON THE PERFORMANCE OF ISOPARAMETRIC ELEMENTS
}

\author{
NAM-SUA LEE* AND KLAUS-JÜRGEN BATHE ${ }^{\dagger}$ \\ Department of Mechanical Engineering, Massachusetts Institute of Technology, Cambridge, \\ MA 02139, U.S.A.
}

\begin{abstract}
SUMMARY
We discuss the effects of element distortions on the performance of displacement-based isoparametric quadrilateral finite elements. Suitable example problems for both the Lagrangian and the serendipity types of elements are used to show numerically the effects of element distortions. The effects of angular and curved-edge distortions are evaluated analytically in terms of the abilities of the elements to represent exactly various polynomial displacement fields. We then solve a plane stress problem adaptively to demonstrate the effect of element distortions on the accuracy of the stress distribution. The conclusion is that the Lagrangian elements are not affected by angular distortions and are therefore the more reliable elements for general use.
\end{abstract}

\section{INTRODUCTION}

The stiffening of isoparametric elements when distorted was observed by many authors. Stricklin et $a .^{1}$ presented some results for a cantilever beam modelled with distorted and undistorted elements and showed that the 8-node isoparametric elements stiffened and performed very badly when distorted. This conclusion was supported by Bäcklund, ${ }^{2}$ Gifford ${ }^{3}$ and Burrows. ${ }^{4}$ Gifford gave additional results for the 12-node isoparametric element and suggested that element distortions should be limited to less than $45^{\circ}$ in order to obtain acceptable results.

It should, however, be noted that these authors all used the serendipity type of isoparametric elements -i.e. those without interior nodes--in their numerical experiments. For comparison, we performed similar experiments using 9-node and 16-node Lagrangian-type elements. Our results are compiled together with those of Stricklin et al., ${ }^{1}$ Bäcklund $^{2}$ and Gifford ${ }^{3}$ in Table I.

Comparing the results of Table I, we immediately notice the superiority of the Lagrangian-type elements when distorted. Note particularly that while the 8-node and 12-node serendipity elements perform very badly using Mesh 4, the performance of the 16-node Lagrangian element is little affected by the severe distortion. The exact solution to the problem is slightly different from that for a simple cantilever beam with an end load because the cross-section is completely fixed at the left end; hence, even the 16-node element models cannot solve the problem exactly.

It is clear that the elements considered in Table I have been in extensive use for over two decades and a number of studies on their performance when used in distorted shapes have been published. ${ }^{1-11}$ However, there is still a need for more insight and clearer guidelines on the use of

\footnotetext{
*Graduate Student

${ }^{\dagger}$ Professor of Mechanical Engineering
} 


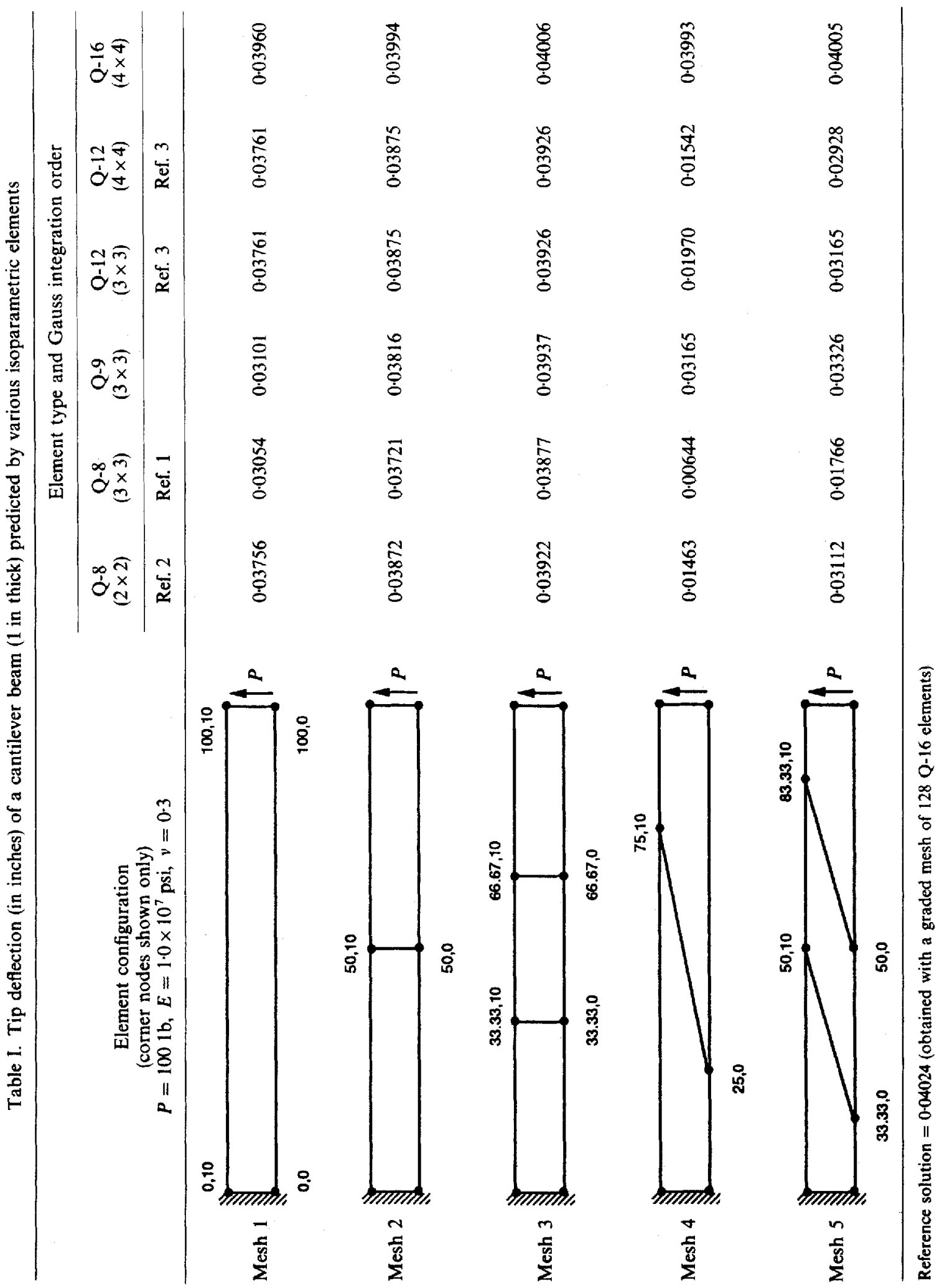


distorted isoparametric elements. The objective of this paper is to present a systematic study with numerical and analytical results on the performance of the elements when they are used in distorted shapes and summarize the findings that have practical consequences.

In the paper, we focus our attention on the types of element distortions that occur most frequently in practice and that affect the performance of the elements. To aid our study, we refer to a very simple classification of element distortions based on the shape of the element and the location of its nodes. (See References $8-10$ for other criteria used in classifying element distortions.)

We consider a square element with evenly spaced nodes to be the undistorted element. Comparing with this configuration, we may identify the following basic types of distortions:

1. aspect-ratio and parallelogram distortions,

2. unevenly-spaced-nodes distortion,

3. angular distortion, and

4. curved-edge distortion.

These distortions are illustrated in Figure 1. Any element distortion can generally be considered to be composed of some or all of the above types of distortions. We may also distinguish between distortions that are present in the undeformed finite element model and distortions that are created by large deformations. The latter type occur only in non-linear analysis with large strain effects.

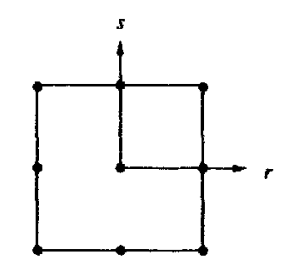

undistorted configuration

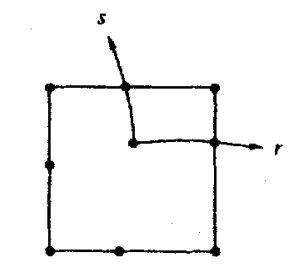

unevenly-spaced-nodes distortion

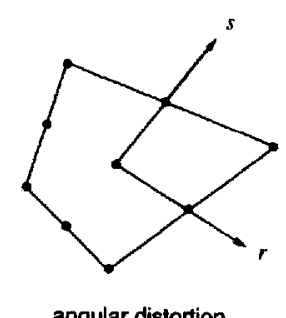

angular dislortion
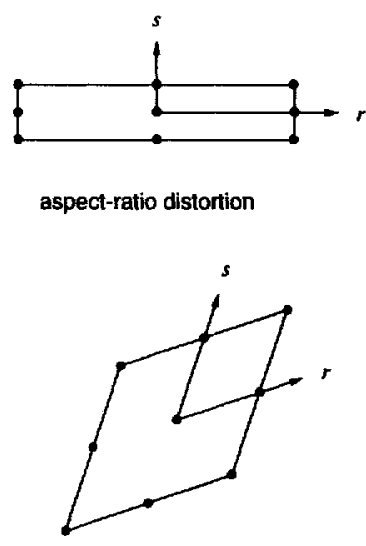

parallelogram distortion

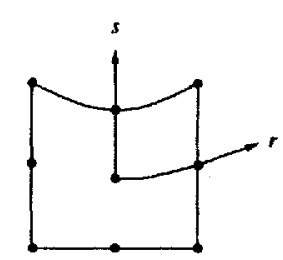

curvededge distortion

Figure 1. Classification of element distortions 
Let us assume that the element is used in the analysis of a two-dimensional continuum. Then the only effect of aspect-ratio or parallelogram distortions is to reduce the predictive capabilities of the overall finite element model in the direction that has fewer elements per unit length. The consequences of using such elements are quite easy to predict. Since aspect-ratio and parallelogram distortions do not affect the element performance (which is our main concern), we shall not address such distortions in this paper.

Unevenly-spaced-nodes distortions are usually created intentionally to achieve special effects, e.g. $1 / \sqrt{r}$ stress singularity effects in fracture mechanics analysis. Elements with such distortions should not be used in the analyses of smooth problems, since they are also not spatially isotropic. In non-linear analysis, large deformations with large strain variations within the element will be necessary to create a significant unevenly-spaced-nodes distortion. This type of element distortion may result in various complex effects and will not be considered in the following sections; all elements used will always have their interior and edge nodes evenly spaced.

Angular distortions occur most frequently in practice and are usually unavoidable. They are present, for example,

1. in the transition regions from coarser to finer meshes,

2. next to complicated geometrical boundaries,

3. in meshes generated by automatic mesh generators, and

4. in meshes created by adaptive algorithms.

In non-linear analysis, significant angular distortions are frequently generated due to varying shear strains in materials that are weak in shear (compared to their bulk stiffness).

Curved-edge distortions also occur very frequently in practice. For example, to model a curved boundary more correctly, the edges of the elements that form the boundary have to be curved. In non-linear analysis, an initially straight edge may become curved if deformation causes strain variations within the element to become large.

In the following sections, we focus our attention on angular and curved-edge distortions. In Section 2, we present and solve two demonstrative problems to study the effect of element distortions on the accuracy of finite element results. In Section 3, we examine various elements for their global polynomial basis functions to see which of these basis functions are retained exactly when the elements become distorted by angular and curved-edge distortions. The case of the 8- and 9-node isoparametric elements subjected to angular distortions was already considered by Zienkiewicz and Taylor ${ }^{12}$ and we merely include these results here for completeness of our presentation.

In Section 4, a plane stress problem is solved adaptively and the effect of using distorted elements in such a scheme is demonstrated. We conclude the paper in Section 5 with several important practical implications.

\section{DEMONSTRATIVE EXAMPLE PROBLEMS}

The difference between the 9-node element results and the reference solution documented in Table $I$ is to some degree due to the inherent inability of this element to represent a cubic displacement field. The effects of distortions, in that particular problem solution, accentuate that inability. In order to separate such effects from the actual effects of element distortions, we consider the following example problems that can be solved exactly by a single element in its undistorted configuration. By solving these problems with distorted elements, we are able to identify directly the effects of element distortions, since the errors in solving these problems are due only to element distortions. 


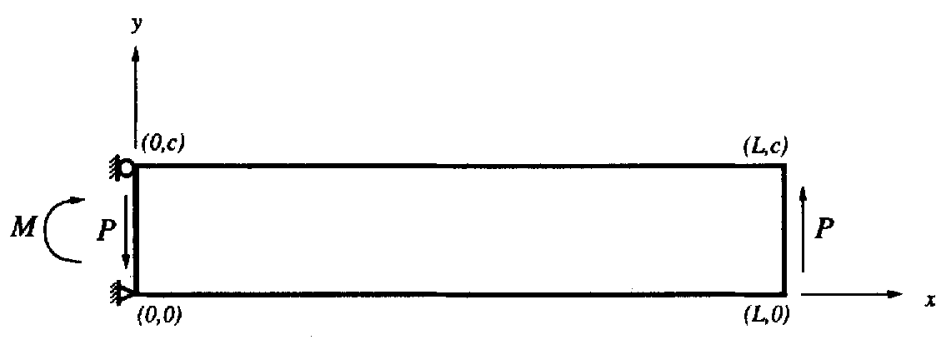

$$
\begin{aligned}
& \text { Young's modulus } E=1.0 \times 10^{7} \\
& \text { Poisson's ratio } \nu=0.3 \\
& \text { plane stress condition } \\
& \text { thickness } t=1.0 \\
& P=\frac{20 c^{2}}{L} \quad \text { distributed as } f_{y}=\frac{120 y}{L}-\frac{120 y^{2}}{c L} \\
& M=20 c^{2} \quad \text { distributed as } f_{x}=\frac{240 y}{c}-120
\end{aligned}
$$

Figure 2. Linear bending problem for cubic elements

\subsection{A problem for cubic elements}

For elements that have complete polynomial basis functions of at least degree 3 (12- and 16-node elements, for example), we use the linear bending-moment problem described in Figure 2 to demonstrate the effects of element distortions. Note that this problem is different from the problem of Table I at the 'fixed' boundary. Here, in order to allow the 2-D plane stress problem to behave like a beam, we have set the edge nodes free and have prescribed the correct shear and bending reactions. Of course, the shear force and bending moment are modelled in the 2-D elasticity problem as distributed forces $f_{x}$ and $f_{y}$ and are in the finite element model represented by consistent nodal point forces. With these details, the exact analytical solution to the problem in Figure 2 is given by

$$
\begin{aligned}
\sigma_{x x}= & \frac{240}{c L} x y-\frac{120}{L} x-\frac{240}{c} y+120 \\
\sigma_{y y}= & 0 \\
\tau_{x y}= & -\frac{120}{c L} y^{2}+\frac{120}{L} y \\
u= & \left(\frac{120}{c L} x^{2} y-\frac{92}{c L} y^{3}-\frac{60}{L} x^{2}-\frac{240}{c} x y+\frac{138}{L} y^{2}+120 x-\frac{46 c}{L} y\right) / E \\
v= & \left(-\frac{40}{c L} x^{3}-\frac{36}{c L} x y^{2}+\frac{120}{c} x^{2}+\frac{36}{L} x y+\frac{36}{c} y^{2}+\frac{46 c}{L} x-36 y\right) / E \\
& \quad \text { for } 0 \leqslant x \leqslant L \text { and } 0 \leqslant y \leqslant c
\end{aligned}
$$

where $u$ and $v$ are, respectively, the $x$-and $y$-displacements. (Note that this 'structural problem' is merely used to evaluate the distortion sensitivity of the elements for the considered strain variation. It, of course, does not imply that the elements will be used in the analysis of beams.) 

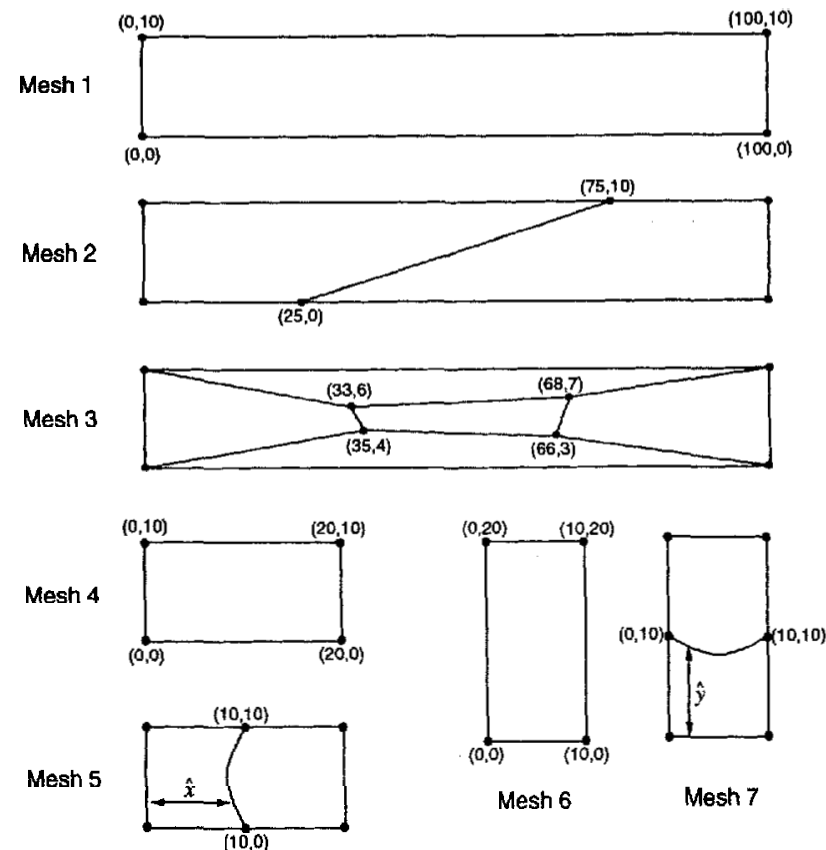

$$
\hat{x}(y)=10-8\left(\frac{y}{10}\right)\left(1-\frac{y}{10}\right) \quad \hat{y}(x)=10-8\left(\frac{x}{10}\right)\left(1-\frac{x}{10}\right)
$$

Figure 3. Meshes used in the solutions of the demonstrative problems (Only corner nodes are shown.)

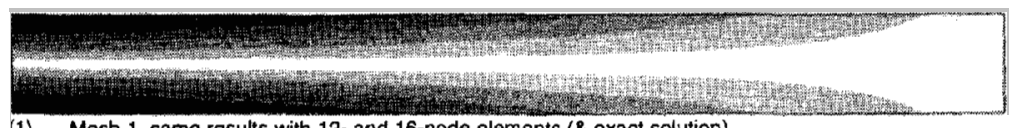

(1) Mesh 1, same results with 12- and 16-node elements (\& exact solution)

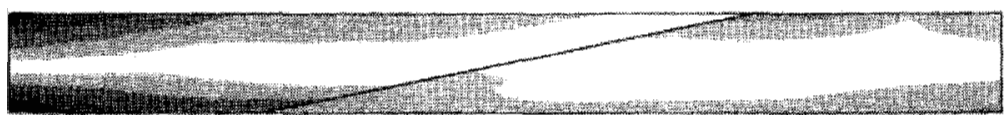

(2A) Mesh 2, 12-node elements

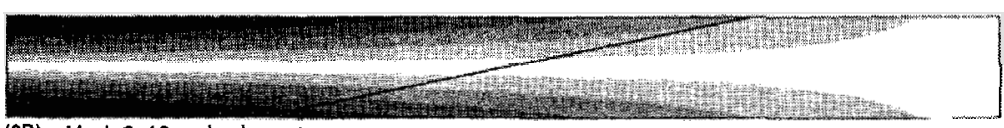

(2B) Mesh 2, 16-node elements

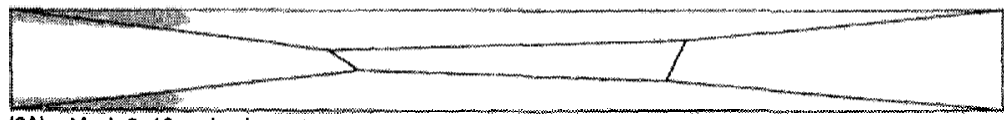

(3A) Mesh 3, 12-node elements

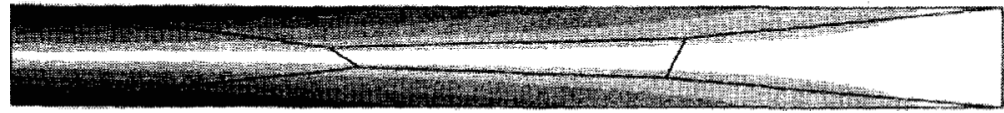

(3B) Mesh 3, 16-node elements

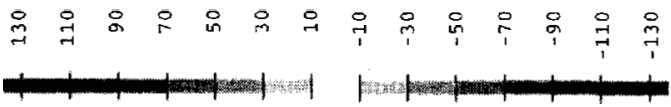

Figure 4. Stress band plots of longitudinal stresses $\left(\sigma_{x x}\right)$ in linear bending-moment problem using Meshes 1,2 and 3 
In order to evaluate the sensitivity of the 12-node and 16-node isoparametric elements to distortions, we solve the above problem using seven different meshes, as shown in Figure 3. Meshes 1, 4 and 6 all consist of one undistorted element; these results are used as the basis for comparison. Meshes 2 and 3 consist of elements having severe angular distortions, while Meshes 5 and 7 consist of elements that have one of their edges curved (a reasonable amount) in the $y$-direction (Mesh 5) or in the $x$-direction (Mesh 7).

The resulting finite element solutions are compared numerically at several representative points in Table II. The overall solutions for the longitudinal stress $\left(\sigma_{x x}\right)$ are also compared by looking at the Sussman-Bathe stress band plots ${ }^{13}$ in Figures 4 and 5 . Note that all stresses presented here are actual finite element stresses (i.e. no stress smoothing has been applied) and that full numerical integration has been used for all element formulations.

The results show that the 16-node Lagrangian element is not affected by angular distortions (no matter how severe they may be) whereas the 12-node element performs poorly when subjected to such distortions. The 12- and 16-node elements performed reasonably well in the case of curved-edge distortions.

\subsection{A problem for quadratic elements}

For elements that have complete polynomial basis functions of at least degree 2 (8-, 9-, 12- and 16-node elements), we use the constant-bending-moment problem shown in Figure 6 to demon-

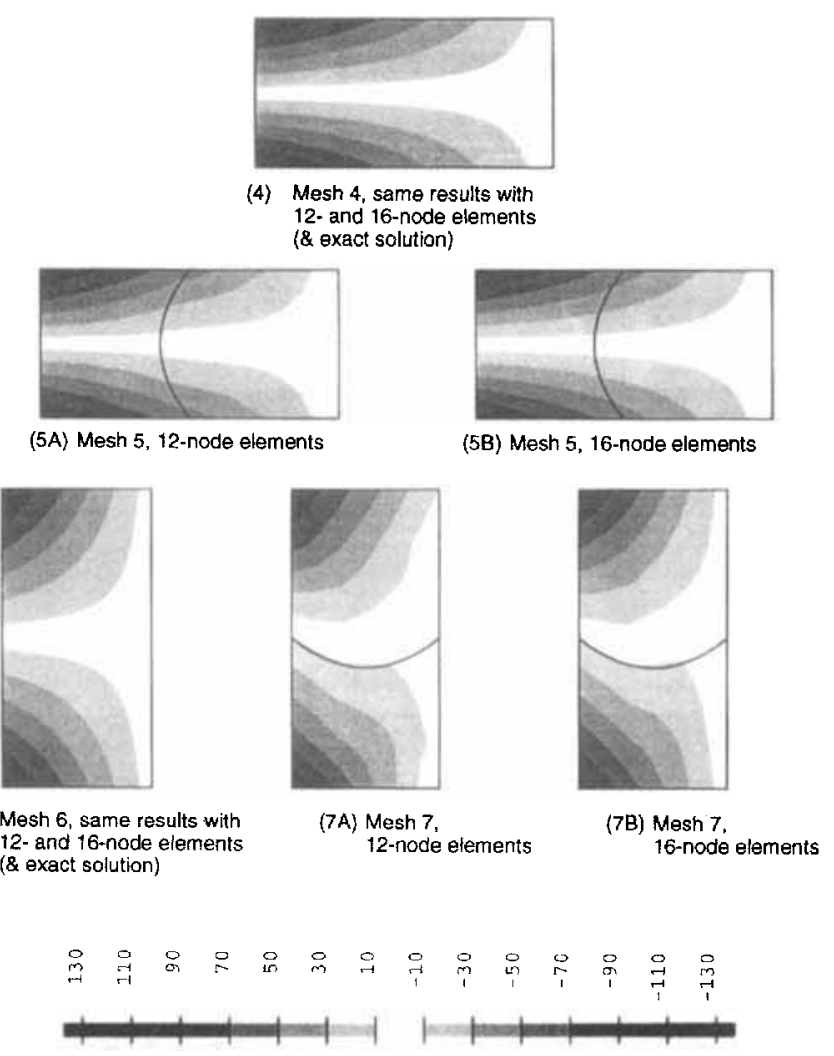

Figure 5. Stress band plots of longitudinal stresses $\left(\sigma_{x x}\right)$ in linear bending-moment problem using Meshes $4,5,6$ and 7 


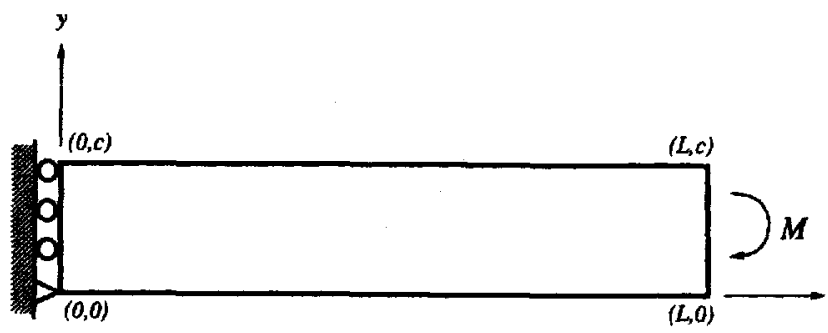

Young's modulus $E=1.0 \times 10^{7}$

Poisson's ratio $\nu=0.3$

plane stress condition

thickness $t=1.0$

$M=20 c^{2} \quad$ distributed as $f_{x}=\frac{240 y}{c}-120$

Figure 6. Constant-bending-moment problem for quadratic elements

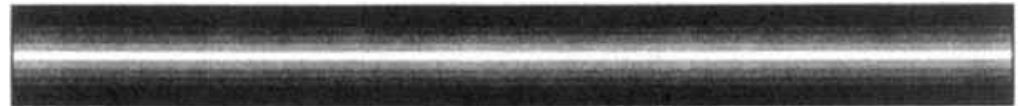

(1) Mesh 1, same results with 8-, 9-, 12- and 16-node elements (\& exact solution)

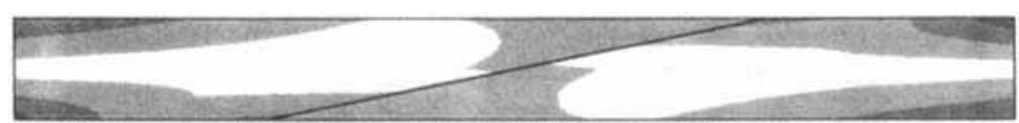

(2A) Mesh 2, 8-node elements

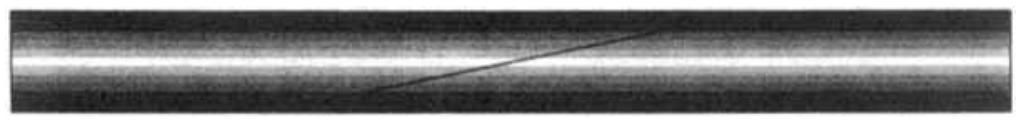

(2B) Mesh 2, 9-node elements

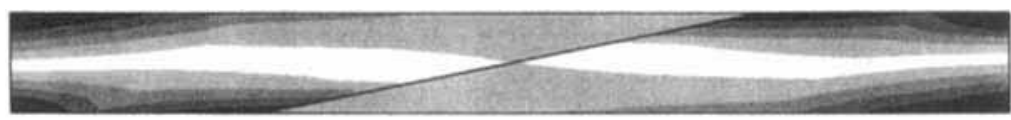

(2C) Mesh 2, 12-node elements

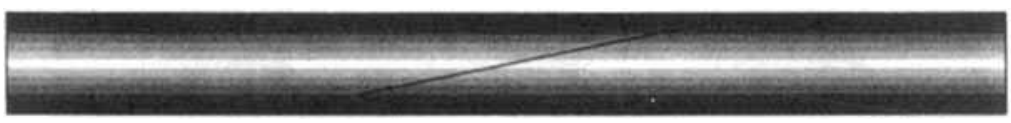

(20) Mesh 2, 16-node elements

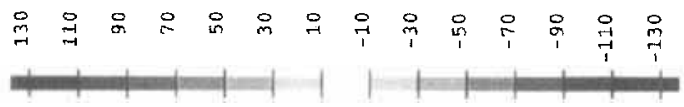

Figure 7. Stress band plots of longitudinal stresses $\left(\sigma_{x x}\right)$ in constant-bending-moment problem using Meshes 1 and 2 
Table II. Numerical results at selected locations for linear bending-moment problem

\begin{tabular}{|c|c|c|c|c|}
\hline & & $\begin{array}{l}\text { 12-node } \\
\text { element }\end{array}$ & $\begin{array}{l}\text { 16-node } \\
\text { element }\end{array}$ & $\begin{array}{c}\text { Exact } \\
\text { solution }\end{array}$ \\
\hline Mesh 1 & $\begin{array}{c}\sigma_{x}(0,10) \\
\sigma_{x}(0,0) \\
\tau_{x y}(0,5) \\
v(100,0) \times 10^{3}\end{array}$ & $\begin{array}{r}-120.0 \\
120.0 \\
3.000 \\
8.046\end{array}$ & $\begin{array}{r}-120.0 \\
120.0 \\
3.000 \\
8.046\end{array}$ & $\begin{array}{r}-120 \cdot 0 \\
120 \cdot 0 \\
3 \cdot 000 \\
8 \cdot 046\end{array}$ \\
\hline Mesh 2 & $\begin{array}{c}\sigma_{x}(0,10) \\
\sigma_{x}(0,0) \\
\tau_{x y}(0,5) \\
v(100,0) \times 10^{3}\end{array}$ & $\begin{array}{c}-89.2 \\
131.4 \\
21.69 \\
3.444\end{array}$ & $\begin{array}{r}-120.0 \\
120.0 \\
3.000 \\
8.046\end{array}$ & $\begin{array}{r}-120 \cdot 0 \\
120 \cdot 0 \\
3 \cdot 000 \\
8 \cdot 046\end{array}$ \\
\hline Mesh 3 & $\begin{array}{c}\sigma_{x}(0+, 10) \\
\sigma_{x}(0,10-) \\
\sigma_{x}(0,0+) \\
\sigma_{x}(0+, 0) \\
\tau_{x y}(0,5) \\
v(100,0) \times 10^{3}\end{array}$ & $\begin{array}{c}-23 \cdot 6 \\
-14.4 \\
13 \cdot 6 \\
22.5 \\
25 \cdot 15 \\
0.585\end{array}$ & $\begin{array}{r}-120.0 \\
-120 \cdot 0 \\
120 \cdot 0 \\
120 \cdot 0 \\
3.000 \\
8.046\end{array}$ & $\begin{array}{r}-120 \cdot 0 \\
-120 \cdot 0 \\
120 \cdot 0 \\
120.0 \\
3.000 \\
8.046\end{array}$ \\
\hline Mesh 4 & $\begin{array}{c}\sigma_{x}(0,10) \\
\sigma_{x}(0,0) \\
\tau_{x y}(0,5) \\
v(20,0) \times 10^{4}\end{array}$ & $\begin{array}{c}-120 \cdot 0 \\
120 \cdot 0 \\
15 \cdot 00 \\
3.660\end{array}$ & $\begin{array}{c}-120 \cdot 0 \\
120 \cdot 0 \\
15 \cdot 00 \\
3 \cdot 660\end{array}$ & $\begin{array}{c}-120 \cdot 0 \\
120 \cdot 0 \\
15 \cdot 00 \\
3.660\end{array}$ \\
\hline Mesh 5 & $\begin{array}{c}\sigma_{x}(0,10) \\
\sigma_{x}(0,0) \\
\tau_{x y}(0,5) \\
v(20,0) \times 10^{4}\end{array}$ & $\begin{array}{c}-112.0 \\
112.0 \\
13.97 \\
3.534\end{array}$ & $\begin{array}{c}-120 \cdot 4 \\
120 \cdot 4 \\
15 \cdot 09 \\
3.659\end{array}$ & $\begin{array}{c}-120 \cdot 0 \\
120 \cdot 0 \\
15 \cdot 00 \\
3 \cdot 660\end{array}$ \\
\hline Mesh 6 & $\begin{array}{c}\sigma_{x}(0,20) \\
\sigma_{x}(0,0) \\
\tau_{x y}(0,10) \\
v(10,0) \times 10^{4}\end{array}$ & $\begin{array}{c}-120 \cdot 0 \\
120 \cdot 0 \\
60 \cdot 00 \\
1 \cdot 320\end{array}$ & $\begin{array}{c}-120 \cdot 0 \\
120-0 \\
60-00 \\
1 \cdot 320\end{array}$ & $\begin{array}{c}-120 \cdot 0 \\
120 \cdot 0 \\
60 \cdot 00 \\
1.320\end{array}$ \\
\hline Mesh 7 & $\begin{array}{c}\sigma_{x}(0,20) \\
\sigma_{x}(0,0) \\
\tau_{x y}(0,10+) \\
\tau_{x y}(0,10-) \\
v(10,0) \times 10^{4}\end{array}$ & $\begin{array}{c}-105.5 \\
134.7 \\
57.63 \\
59.04 \\
1.300\end{array}$ & $\begin{array}{c}-119 \cdot 8 \\
120 \cdot 6 \\
61 \cdot 39 \\
60 \cdot 38 \\
1.314\end{array}$ & $\begin{array}{c}-120 \cdot 0 \\
120 \cdot 0 \\
60 \cdot 00 \\
60 \cdot 00 \\
1.320\end{array}$ \\
\hline
\end{tabular}

strate the effects of element distortions. The exact analytical solution in this case is given by

$$
\begin{aligned}
\sigma_{x x} & =\frac{240}{c} y-120 \\
\sigma_{y y} & =0 \\
\tau_{x y} & =0 \\
u & =\left(\frac{240}{c} x y-120 x\right) / E \\
v & =\left(-\frac{120}{c} x^{2}-\frac{36}{c} y^{2}+36 y\right) / E \text { for } 0 \leqslant x \leqslant L \text { and } 0 \leqslant y \leqslant c .
\end{aligned}
$$




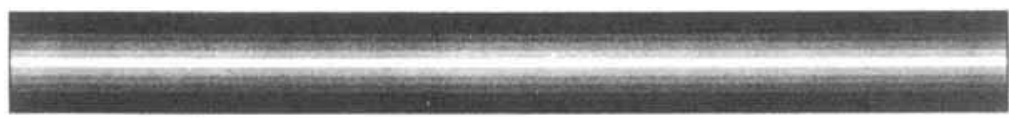

(1) Exact solution

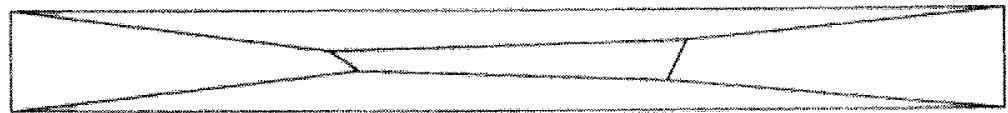

(3A) Mesh 3, 8-node elements

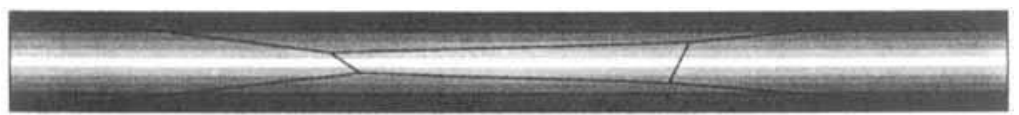

(3B) Mesh 3, 9-node elements

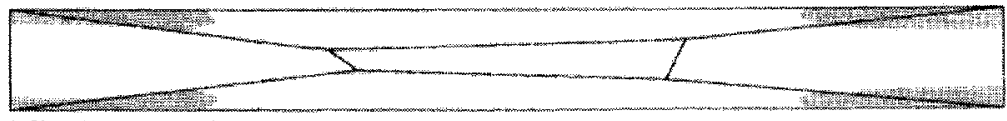

(3C) Mesh 3, 12-node elements

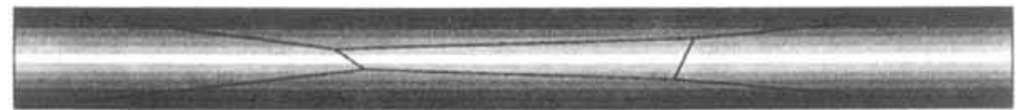

(3D) Mesh 3, 16-node elements

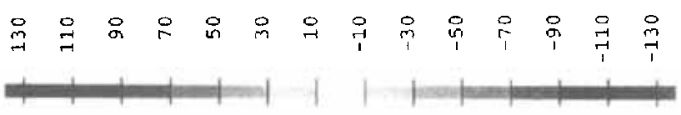

Figure 8. Stress band plots of longitudinal stresses $\left(\sigma_{x x}\right)$ in constant-bending-moment problem using Mesh 3

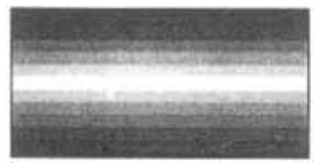

(4) Mesh 4, same results with B-, 9-, 12- and 16-node elements (\& exact solution)

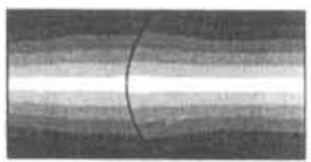

(5A) Mesh 5, B-node elements

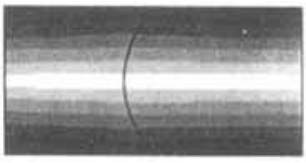

(5C) Mesh 5, 12-node elements

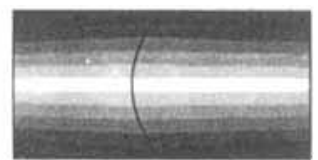

(5B) Mesh 5, 9-node elements

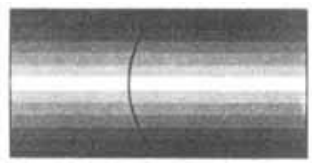

(5D) Mesh 5, 16-node elements

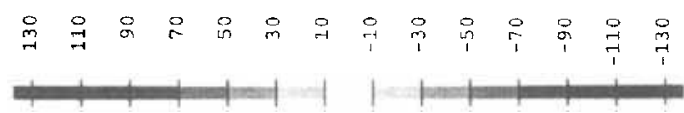

Figure 9. Stress band plots of longitudinal stresses $\left(\sigma_{x x}\right)$ in constant-bending-moment problem using Meshes 4 and 5 


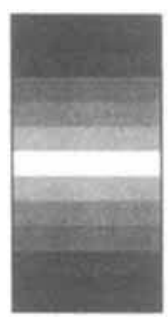

(6) Mesh 6, same results with 8-, 9-, 12- and 16-node elements (\& exact solution)

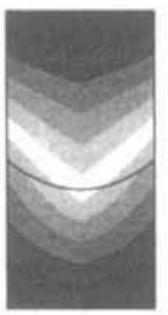

(7A) Mesh 7, 8-node elements

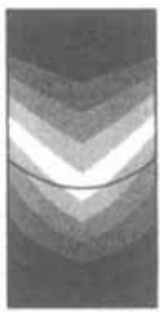

(7B) Mesh 7 9-node elements

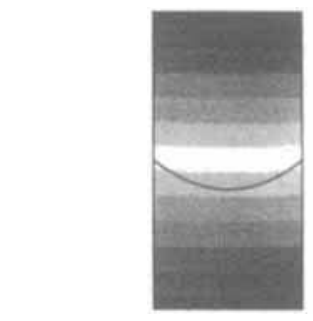

(7C) Mesh 7, 12-node elements

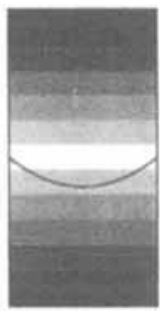

(7D) Mesh 7, 16-node elements

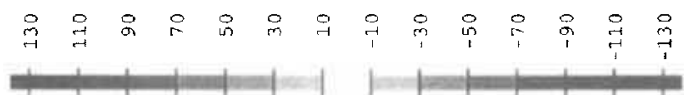

Figure 10. Stress band plots of longitudinal stresses $\left(\sigma_{x x}\right)$ in constant-bending-moment problem using Meshes 6 and 7

We solve this problem using 8-node, 9-node, 12-node and 16-node isoparametric elements and the meshes of Figure 3. The numerical results are tabulated in Table III and the stress band plots are given in Figures 7-10.

The results show that both the 9-node and 16-node Lagrangian elements are not affected by angular distortions, no matter how severe they may be. The 8- and 12-node serendipity elements, on the other hand, are badly affected by angular distortions. We also see that the 8- and 9-node elements are significantly affected by the curved-edge distortions, whereas the 12-node element results differ only slightly from the exact solution and the 16-node element results are excellent.

\section{ANALYSIS OF THE ELEMENTS}

Consider the 16-node Lagrangian element shown in Figure 11. We use $r$ and $s$ for the local co-ordinates of this isoparametric element. The element displacements $-u$ in the $x$-direction and $v$ in the $y$-direction-are then given by

and

$$
u=\sum_{i=1}^{16} h_{i}(r, s) \hat{u}_{i}
$$

$$
v=\sum_{i=1}^{16} h_{i}(r, s) \hat{v}_{i}
$$


Table III. Numerical results at selected locations for constant-bending-moment problem

\begin{tabular}{|c|c|c|c|c|c|c|}
\hline & & $\begin{array}{l}\text { 8-node } \\
\text { element }\end{array}$ & $\begin{array}{l}\text { 9-node } \\
\text { element }\end{array}$ & $\begin{array}{l}\text { 12-node } \\
\text { element }\end{array}$ & $\begin{array}{l}\text { 16-node } \\
\text { element }\end{array}$ & $\begin{array}{l}\text { Exact } \\
\text { solution }\end{array}$ \\
\hline Mesh 1 & $\begin{array}{c}\sigma_{x}(0,10) \\
\sigma_{x}(0,0) \\
v(100,0) \times 10^{3}\end{array}$ & $\begin{array}{c}120 \cdot 0 \\
-120 \cdot 0 \\
-12 \cdot 00\end{array}$ & $\begin{array}{c}120 \cdot 0 \\
-120 \cdot 0 \\
-12.00\end{array}$ & $\begin{array}{c}120 \cdot 0 \\
-120 \cdot 0 \\
-12.00\end{array}$ & $\begin{array}{c}120-0 \\
-120 \cdot 0 \\
-12.00\end{array}$ & $\begin{array}{c}120 \cdot 0 \\
-120 \cdot 0 \\
-12.00\end{array}$ \\
\hline Mesh 2 & $\begin{array}{c}\sigma_{x}(0,10) \\
\sigma_{x}(0,0) \\
v(100,0) \times 10^{3}\end{array}$ & $\begin{array}{c}56 \cdot 5 \\
-74.9 \\
-2.33\end{array}$ & $\begin{array}{c}120 \cdot 0 \\
-120 \cdot 0 \\
-12 \cdot 00\end{array}$ & $\begin{aligned} & 125 \cdot 5 \\
- & 145 \cdot 5 \\
- & -5 \cdot 18\end{aligned}$ & $\begin{array}{c}120.0 \\
-120.0 \\
-12.00\end{array}$ & $\begin{array}{c}120 \cdot 0 \\
-120 \cdot 0 \\
-12 \cdot 00\end{array}$ \\
\hline Mesh 3 & $\begin{array}{c}\sigma_{x}(0+, 10) \\
\sigma_{x}(0,10-) \\
\sigma_{x}(0,0+) \\
\sigma_{x}(0+, 0) \\
v(100,0) \times 10^{3}\end{array}$ & $\begin{array}{c}13 \cdot 8 \\
5 \cdot 2 \\
-5 \cdot 8 \\
-14 \cdot 5 \\
-0.48\end{array}$ & $\begin{array}{r}120 \cdot 0 \\
120 \cdot 0 \\
-120 \cdot 0 \\
-120 \cdot 0 \\
-12 \cdot 00\end{array}$ & $\begin{array}{c}29 \cdot 4 \\
14 \cdot 0 \\
-13 \cdot 1 \\
-28 \cdot 5 \\
-0 \cdot 69\end{array}$ & $\begin{array}{r}120.0 \\
120.0 \\
-120.0 \\
-120.0 \\
-12.00\end{array}$ & $\begin{array}{c}120 \cdot 0 \\
120 \cdot 0 \\
-120 \cdot 0 \\
-120 \cdot 0 \\
-12 \cdot 00\end{array}$ \\
\hline Mesh 4 & $\begin{array}{c}\sigma_{x}(0,10) \\
\sigma_{x}(0,0) \\
v(20,0) \times 10^{4}\end{array}$ & $\begin{array}{c}120 \cdot 0 \\
-120 \cdot 0 \\
-4.800\end{array}$ & $\begin{array}{c}120.0 \\
-120 \cdot 0 \\
-4.800\end{array}$ & $\begin{array}{c}120 \cdot 0 \\
-120 \cdot 0 \\
-4 \cdot 800\end{array}$ & $\begin{array}{c}120.0 \\
-1200 \\
-4 \cdot 800\end{array}$ & $\begin{array}{c}1200 \\
-1200 \\
-4 \cdot 800\end{array}$ \\
\hline Mesh 5 & $\begin{array}{c}\sigma_{x}(0,10) \\
\sigma_{x}(0,0) \\
v(20,0) \times 10^{4}\end{array}$ & $\begin{array}{c}120 \cdot 2 \\
-120 \cdot 2 \\
-4 \cdot 412\end{array}$ & $\begin{array}{c}111.9 \\
-111.9 \\
-4.411\end{array}$ & $\begin{array}{c}120 \cdot 9 \\
-120 \cdot 9 \\
-4 \cdot 774\end{array}$ & $\begin{array}{c}120.6 \\
-120 \cdot 6 \\
-4.795\end{array}$ & $\begin{array}{c}120 \cdot 0 \\
-120 \cdot 0 \\
-4 \cdot 800\end{array}$ \\
\hline Mesh 6 & $\begin{array}{c}\sigma_{x}(0,20) \\
\sigma_{x}(0,0) \\
v(10,0) \times 10^{5}\end{array}$ & $\begin{array}{c}120.0 \\
-120 \cdot 0 \\
-6.000\end{array}$ & $\begin{array}{c}120.0 \\
-120.0 \\
-6.000\end{array}$ & $\begin{array}{l}120.0 \\
-120.0 \\
-6.000\end{array}$ & $\begin{array}{c}120.0 \\
-120.0 \\
-6.000\end{array}$ & $\begin{array}{c}120.0 \\
-120.0 \\
-6.000\end{array}$ \\
\hline Mesh 7 & $\begin{array}{c}\sigma_{x}(0,20) \\
\sigma_{x}(0,0) \\
\nu(10,0) \times 10^{5}\end{array}$ & $\begin{array}{c}115 \cdot 2 \\
-119 \cdot 9 \\
-6.045\end{array}$ & $\begin{array}{c}115.9 \\
-115.8 \\
-6.022\end{array}$ & $\begin{array}{c}120 \cdot 3 \\
-119 \cdot 1 \\
-5 \cdot 994\end{array}$ & $\begin{array}{c}119.9 \\
-119.9 \\
-6.003\end{array}$ & $\begin{array}{c}120.0 \\
-120.0 \\
-6.000\end{array}$ \\
\hline
\end{tabular}

where $\hat{u}_{i}$ and $\hat{v}_{i}$ are, respectively, the $x$-and $y$-displacement at node $i$ of the element, $h_{i}(r, s)$ are the interpolation functions defined in the $(r, s)$ local co-ordinate system, $-1 \leqslant r, s \leqslant+1$, and $h_{i}$ is unity at node $i$ and is zero at all other nodes. ${ }^{14}$

Consider the $x$-displacement as given by equation (1). By expanding this equation and collecting terms in $r, s, r s$, etc., we can easily obtain

$$
\begin{aligned}
u= & \alpha_{1}+\alpha_{2} r+\alpha_{3} s+\alpha_{4} r^{2}+\alpha_{5} r s+\alpha_{6} s^{2}+\alpha_{7} r^{3}+\alpha_{8} r^{2} s+\alpha_{9} r s^{2}+\alpha_{10} s^{3} \\
& +\alpha_{11} r^{3} s+\alpha_{12} r^{2} s^{2}+\alpha_{13} r s^{3}+\alpha_{14} r^{3} s^{2}+\alpha_{15} r^{2} s^{3}+\alpha_{16} r^{3} s^{3}
\end{aligned}
$$

where the $\alpha$ 's are independent of $r$ and s. Similarly, for the 12-node serendipity element,

$$
\begin{aligned}
u= & \bar{\alpha}_{1}+\bar{\alpha}_{2} r+\bar{\alpha}_{3} s+\bar{\alpha}_{4} r^{2}+\bar{\alpha}_{5} r s+\bar{\alpha}_{6} s^{2}+\bar{\alpha}_{7} r^{3} \\
& +\bar{\alpha}_{8} r^{2} s+\bar{\alpha}_{9} r s^{2}+\bar{\alpha}_{10} s^{3}+\bar{\alpha}_{11} r^{3} s+\bar{\alpha}_{12} r s^{3}
\end{aligned}
$$

where, again, the $\bar{\alpha}$ 's are independent of $r$ and $s$.

Note that (3) and (4) are the equations for the $x$-displacement field in terms of the local basis functions $\left(1, r, s, r^{2}\right.$, etc.). These equations are valid for any element configuration (regardless of whether the element is distorted or not) since the physical dimensions of the element have not entered into the equations yet.

The frequently overlooked but obvious feature of the isoparametric elements is that each of the global basis functions (e.g. $x^{2} y$ ) is exactly linearly related to only one of the local basis functions 


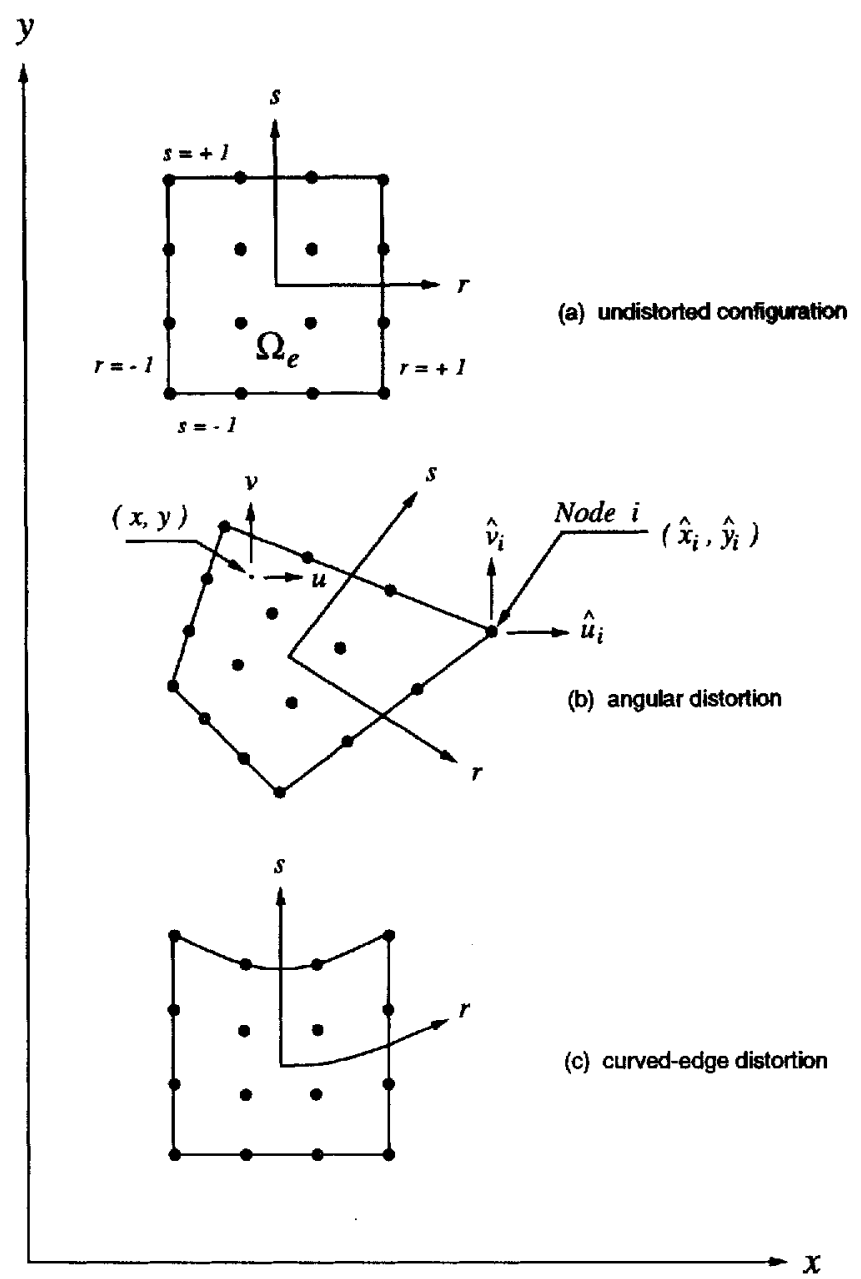

Figure 11. 16-node Lagrangian element in the undistorted and distorted configurations

( $r^{2} s$ in this case) when the elements are rectangular, unrotated, and with all nodes in their 'natural' positions. For example, if the element is rectangular but rotated, the completeness of the global polynomial basis functions ${ }^{\mathbf{1 4}}$ is preserved but the additional global basis functions (beyond those needed for completeness) are changed.

In the isoparametric formulation, the physical co-ordinates of the element are interpolated in the same way as the displacements, i.e. for the 16-node element,

and

$$
x=\sum_{i=1}^{16} h_{i}(r, s) \hat{x}_{i}
$$

$$
y=\sum_{i=1}^{16} h_{i}(r, s) \hat{y}_{i}
$$

where $\hat{x}_{i}$ and $\hat{y}_{i}$ are the physical co-ordinates of node $i$ of the element. 
If the element is of square or rectangular shape and unrotated [see Figure 11(a)] we can solve equations (5) and (6) directly for $r$ and $s$. The solution yields the following linear relationship:

$$
r=\gamma_{1}+\gamma_{2} x
$$

and

$$
s=\delta_{1}+\delta_{2} y
$$

where the $\gamma$ 's and $\delta$ 's are independent of $x$ and $y$, and $(x, y) \in \Omega_{e}$.

By substituting (7) and (8) into (3), we immediately see, as is well known, that under these conditions the 16-node Lagrangian element has the following global basis functions in its solution space:

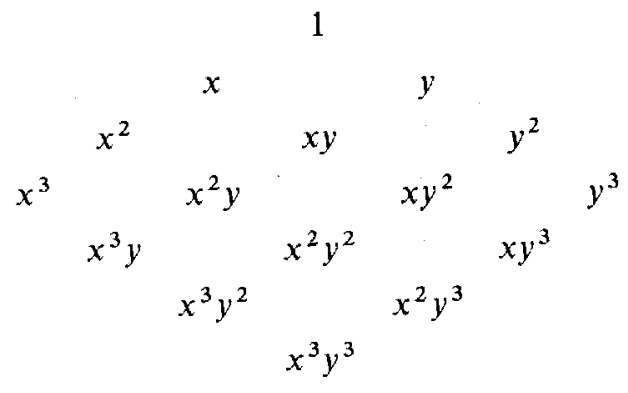

i.e. the 16-node Lagrangian element will be able to solve exactly any polynomial displacement field of the form

where the $c_{i j}$ 's are constants.

$$
\sum_{i=0}^{3} \sum_{j=0}^{3} c_{i j} x^{i} y^{j}
$$

Similarly, as is well-known, the global basis functions for the 12-node serendipity element under these conditions are

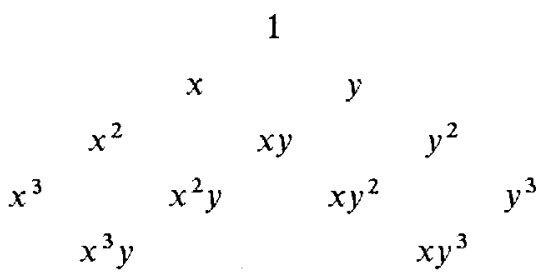

In practice, both the 12- and 16-node elements are considered to be cubic elements because they have complete polynomial basis functions up to degree 3 . In homogeneous meshes of square elements, the order of convergence is given by this degree of completeness in the basis functions, and the same order of convergence in displacements and stresses is applicable for both elements. Thus, when comparing the 16-node element with the 12-node element, one might be misled into thinking that the 'extra' basis functions $\left(x^{2} y^{2}, \ldots, x^{3} y^{3}\right)$ in the 16-node element are not of use. However, as we shall see, these extra basis functions are important because they render the Lagrangian elements insensitive to angular distortions and, therefore, render these elements considerably more robust and powerful than the serendipity or $p$-type elements. ${ }^{15}$

\subsection{Effect of angular distortions}

Consider the angular-distorted 16-node Lagrangian element shown in Figure 11(b). Note that in this configuration the edges are still straight and all interior and edge nodes are still evenly 
spaced in the physical co-ordinate system. This is a bilinear distortion relative to the undistorted configuration and, hence, the physical co-ordinates $(x, y)$ are now related bilinearly to the local co-ordinates $(r, s)$. Under these conditions, equations $(5)$ and $(6)$ reduce to

and

$$
x=\bar{\gamma}_{1}+\bar{\gamma}_{2} r+\bar{\gamma}_{3} s+\bar{\gamma}_{4} r s
$$

$$
y=\bar{\delta}_{1}+\bar{\delta}_{2} r+\bar{\delta}_{3} s+\bar{\delta}_{4} r s
$$

where the $\bar{\gamma}$ 's and $\bar{\delta}$ 's are independent of $r$ and $s$, and $-1 \leqslant r, s \leqslant+1$.

We may proceed from here by solving equations (9) and (10) for $r$ and $s$ in terms of $x$ and $y$, and then substituting the results into equation (3) to obtain the global basis functions for the angulardistorted configuration. A simpler approach, however, is to construct each of the global basis functions using equations (9) and (10) directly. ${ }^{12}$ Thus, we obtain, for example,

$$
\begin{aligned}
x^{3}= & \beta_{1}+\beta_{2} r+\beta_{3} s+\beta_{4} r^{2}+\beta_{5} r s+\beta_{6} s^{2}+\beta_{7} r^{3}+\beta_{8} r^{2} s+\beta_{9} r s^{2}+\beta_{10} s^{3} \\
& +\beta_{11} r^{3} s+\beta_{12} r^{2} s^{2}+\beta_{13} r s^{3}+\beta_{14} r^{3} s^{2}+\beta_{15} r^{2} s^{3}+\beta_{16} r^{3} s^{3}
\end{aligned}
$$

where the $\beta$ 's are independent of $r$ and $s$. Similar expressions are obtained for the other cubic global basis functions $\left(x^{2} y, x y^{2}\right.$ and $\left.y^{3}\right)$ but only with different $\beta$ 's.

Comparing equation (11) with equation (3), we see immediately that the 16-node Lagrangian element in the angular-distorted configuration, regardless of the degree of distortion (but, of course, with all interior angles between 0 and $180^{\circ}$ ), will always have all the third-order global basis functions (and, of course, all the other lower-order basis functions) in its solution space, i.e. the following global basis functions are retained exactly even in the angular-distorted configuration:

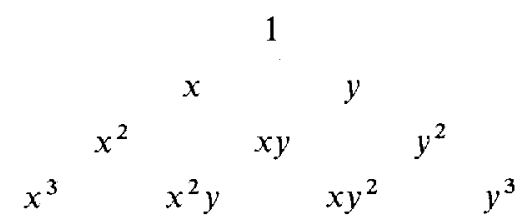

On the other hand, if we compare equation (11) with equation (4), we see that the absence of the 'extra' higher-order terms in the 12-node serendipity element prevents it from retaining any cubic global basis functions exactly.

What about quadratic global basis functions? Can the 12-node serendipity element in its angular-distorted configuration represent quadratic displacement fields exactly?

Again, using equations (9) and (10), we may write the quadratic global basis functions, such as $x^{2}$, in terms of the local co-ordinates as follows:

$$
x^{2}=\bar{\beta}_{1}+\bar{\beta}_{2} r+\bar{\beta}_{3} s+\bar{\beta}_{4} r^{2}+\bar{\beta}_{5} r s+\bar{\beta}_{6} s^{2}+\bar{\beta}_{7} r^{2} s+\bar{\beta}_{8} r s^{2}+\bar{\beta}_{9} r^{2} s^{2}
$$

where the $\bar{\beta}$ 's are independent of $r$ and $s$. Similar expressions are obtained for the other quadratic global basis functions ( $x y$ and $y^{2}$ ) but only with different $\bar{\beta}$ 's.

Comparing equation (12) with equation (4) we can immediately see that the absence of the $r^{2} s^{2}$ term in equation (4) prevents the 12-node serendipity element in its distorted configuration from representing even the quadratic displacement field exactly, i.e. in the distorted configuration, the 12-node serendipity element loses all but the following original global basis functions:

$$
x^{1} y
$$

Of course there are always a total of 12 global basis functions for the 12-node serendipity element. When distorted, the other global basis functions can be obtained (for a particular 
N. S. LEE AND K. J. BATHE

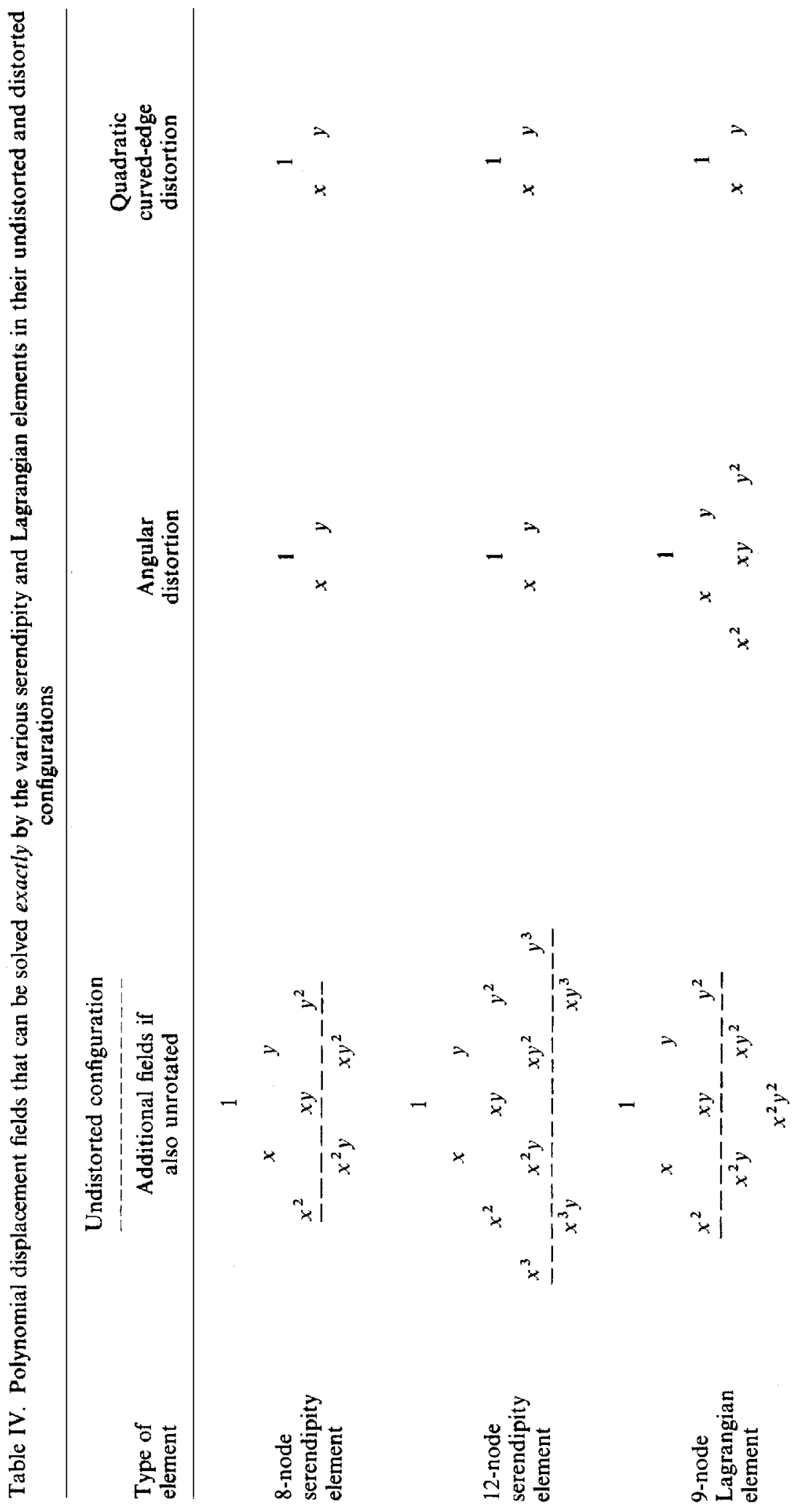


ISOPARAMETRIC ELEMENTS

3569

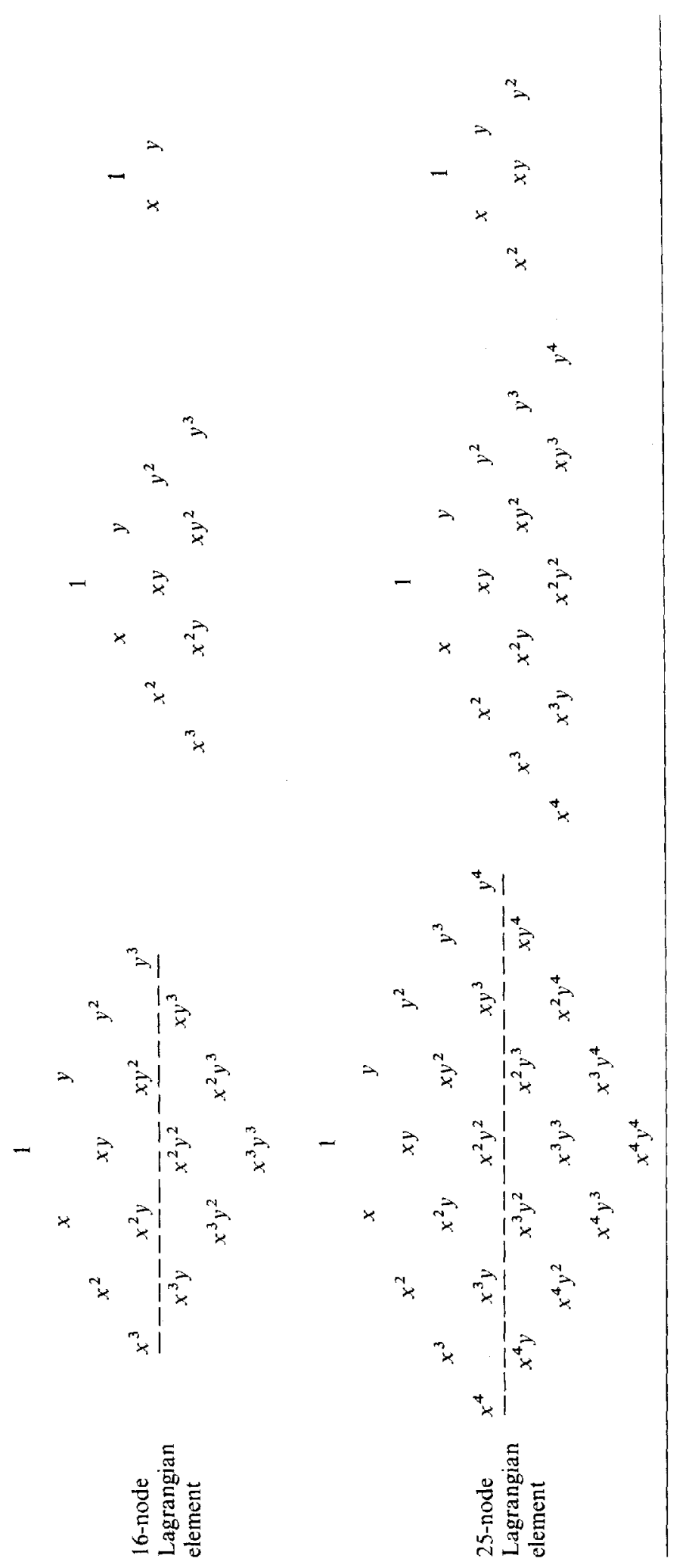


distorted element shape) by solving equations (9) and (10) for $r$ and $s$ in terms of $x$ and $y$, and then substituting the results into equation (4). It is then seen that the other nine global basis functions are expressions of $x$ and $y$ that cannot be linearly combined to reproduce exactly the more fundamental basis functions such as $x^{2}, x y, y^{2}, x^{3}$, etc.

\subsection{Effect of curved-edge distortions}

Consider now the curved-edge distorted 16-node Lagrangian element shown in Figure 11(c). Note that in this configuration all interior and edge nodes are still evenly spaced in the physical co-ordinate system. The simplest form of this type of distortion that can be modelled exactly by the 8-, 9-, 12- and 16-node elements is the quadratic curved-edge distortion considered in Section 2; see Meshes 5 and 7 of Figure 3. Since, in general, sides such as those shown in Meshes 5 and 7 are distorted simultaneously within an element, the physical co-ordinates $(x, y)$ are now related to the local co-ordinates $(r, s)$ in a biquadratic manner. Equations (5) and $(6)$ therefore reduce to

and

$$
x=\tilde{\gamma}_{1}+\tilde{\gamma}_{2} r+\tilde{\gamma}_{3} s+\tilde{\gamma}_{4} r^{2}+\tilde{\gamma}_{5} r s+\tilde{\gamma}_{6} s^{2}+\tilde{\gamma}_{7} r^{2} s+\tilde{\gamma}_{8} r s^{2}+\tilde{\gamma}_{9} r^{2} s^{2}
$$

$$
y=\tilde{\delta}_{1}+\tilde{\delta}_{2} r+\tilde{\delta}_{3} s+\tilde{\delta}_{4} r^{2}+\tilde{\delta}_{5} r s+\tilde{\delta}_{6} s^{2}+\tilde{\delta}_{7} r^{2} s+\tilde{\delta}_{8} r s^{2}+\tilde{\delta}_{9} r^{2} s^{2}
$$

where the $\tilde{\gamma}$ 's and $\tilde{\delta}$ 's are independent of $r$ and $s$, and $-1 \leqslant r, s \leqslant+1$.

Equations (13) and (14) imply that a quadratic global basis function such as $x^{2}$ can be written in terms of $r$ and $s$ as

$$
\begin{aligned}
x^{2}= & \tilde{\beta}_{1}+\tilde{\beta}_{2} r+\tilde{\beta}_{3} s+\tilde{\beta}_{4} r^{2}+\tilde{\beta}_{5} r s+\tilde{\beta}_{6} s^{2}+\tilde{\beta}_{7} r^{3}+\tilde{\beta}_{8} r^{2} s+\tilde{\beta}_{9} r s^{2} \\
& +\tilde{\beta}_{10} s^{3}+\tilde{\beta}_{11} r^{4}+\tilde{\beta}_{12} r^{3} s+\tilde{\beta}_{13} r^{2} s^{2}+\tilde{\beta}_{14} r s^{3}+\tilde{\beta}_{15} s^{4} \\
& +\tilde{\beta}_{16} r^{4} s+\tilde{\beta}_{17} r^{3} s^{2}+\tilde{\beta}_{18} r^{2} s^{3}+\tilde{\beta}_{19} r s^{4}+\tilde{\beta}_{20} r^{4} s^{2} \\
& +\tilde{\beta}_{21} r^{3} s^{3}+\tilde{\beta}_{22} r^{2} s^{4}+\tilde{\beta}_{23} r^{4} s^{3}+\tilde{\beta}_{24} r^{3} s^{4}+\tilde{\beta}_{25} r^{4} s^{4}
\end{aligned}
$$

where the $\tilde{\beta}$ 's are independent of $r$ and $s$. Similar expressions are obtained for the other quadratic global basis functions ( $x y$ and $y^{2}$ ) but only with different $\tilde{\beta}$ 's.

We see immediately from equation (15) that even the 16-node Lagrangian element will not be able to represent exactly a quadratic displacement field whenever it has a curved edge. A 25 -node Lagrangian element will be needed to represent exactly the displacement field of equation (15).

\subsection{Summary of effects of element distortions}

The results of our analyses are summarized in Table IV, and these results explain largely the behaviour of the elements discussed in Section 2. It is important to emphasize that Table IV shows only the terms that are represented exactly by the basis functions. In practice, the elements may perform better because the additional basis functions (beyond those listed in Table IV) may closely approximate those terms needed in the strain representation. For example, the 16-node element when used with a curved-edge distortion in Section 2 clearly performed much better than might be concluded from Table IV (which only shows the constant strain terms as being represented exactly).

\section{A NUMERICAL EXAMPLE}

Consider the problem of a plate with a hole subjected to an in-plane bending moment, as shown in Figure 12. We want to solve this problem accurately for the stress distribution using the finite 


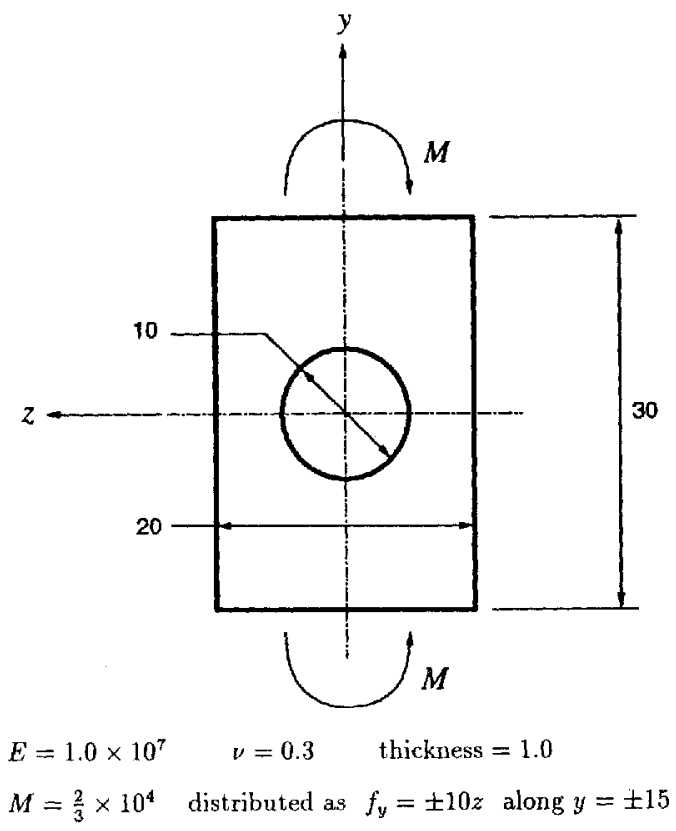

Figure 12. Plate with a central hole subjected to in-plane bending loads

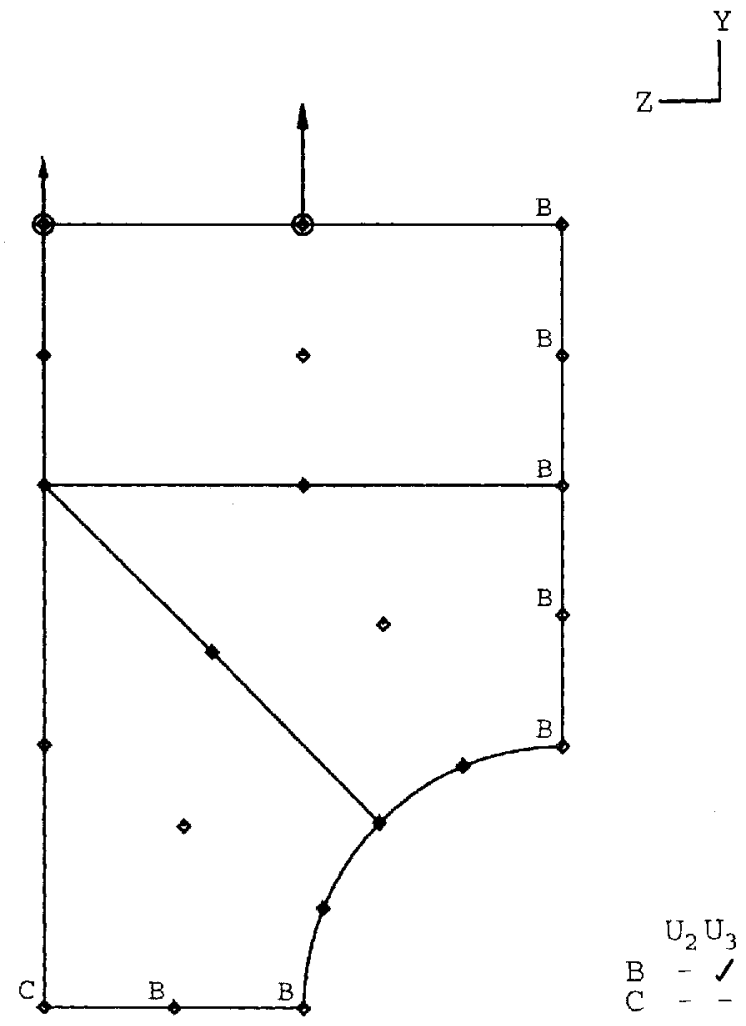

Figure 13. Preliminary finite element model consisting of 9-node Lagrangian elements 

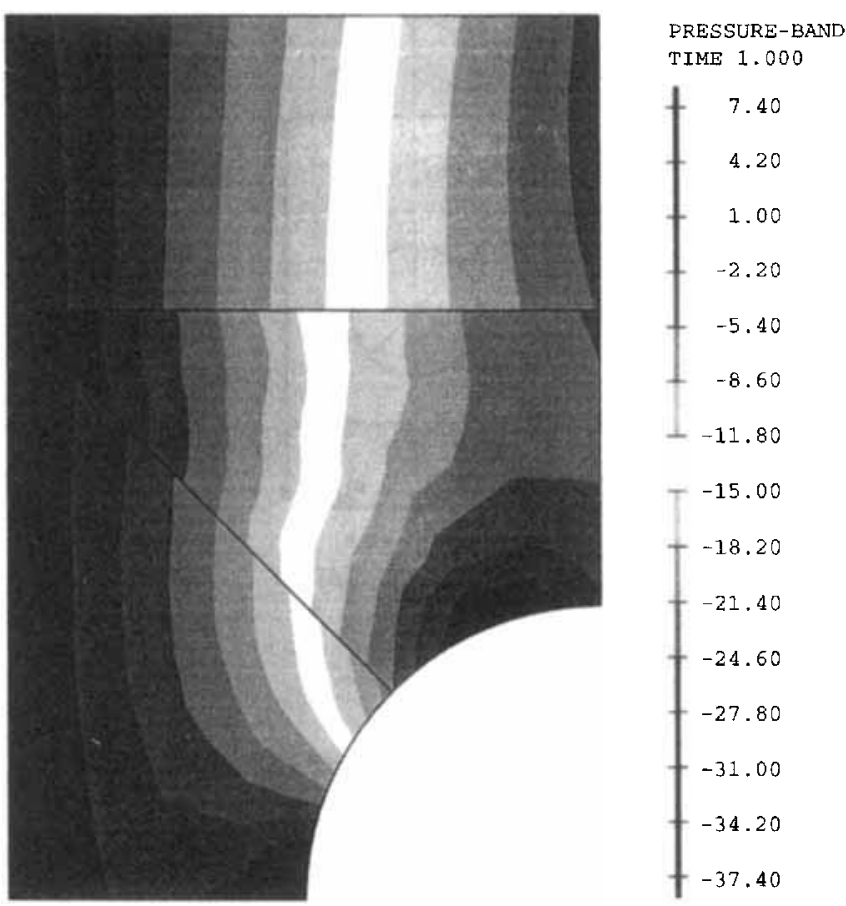

Figure 14. Pressure band plot for the preliminary model

element method. The most effective approach for obtaining a reliable solution is by adaptively modelling the problem until satisfactory results are achieved. ${ }^{16}$ We have used the ADINA finite element system in our study.

We start with a coarse preliminary model consisting of 9-node Lagrangian elements as shown in Figure 13. The pressure band plot of the solution is shown in Figure 14. The waviness and jumps in the pressure bands indicate that the stress distribution results are not very accurate. To improve the finite element model adaptively, we use a pointwise body-force-residual ${ }^{17}$ error indicator to determine where a refinement is needed. The specific form of the error indicator used here is

$$
\text { POINT-BFR }=\frac{V_{\mathrm{c}}}{F_{\mathrm{c}}}\left[\sum_{i=1}^{2}\left\{\left(\sum_{j=1}^{2} \frac{\partial \tau_{i j}^{h}}{\partial x_{j}}\right)+f_{i}^{\mathbf{B}}\right\}^{2}\right]^{1 / 2}
$$

where $V_{\mathrm{c}}=130 \cdot 365$ and $F_{\mathrm{c}}=500$ are, respectively, the characteristic volume and the characteristic applied force, $\tau_{i j}^{h}$ are the predicted finite element stress components, $x_{j}$ are the global co-ordinates, and $f_{i}^{\mathrm{B}}$ are the body force components (which are identically equal to zero in this case).

Figure 15 shows a plot of the pointwise body-force-residual and it is not surprising to notice that refinement is needed near the hole.

We next use an $h-p$ refinement scheme to quickly obtain an accurate solution. Using the ADINA-IN mesh generator, we create a nearly optimal mesh as dictated by the error indicator. 

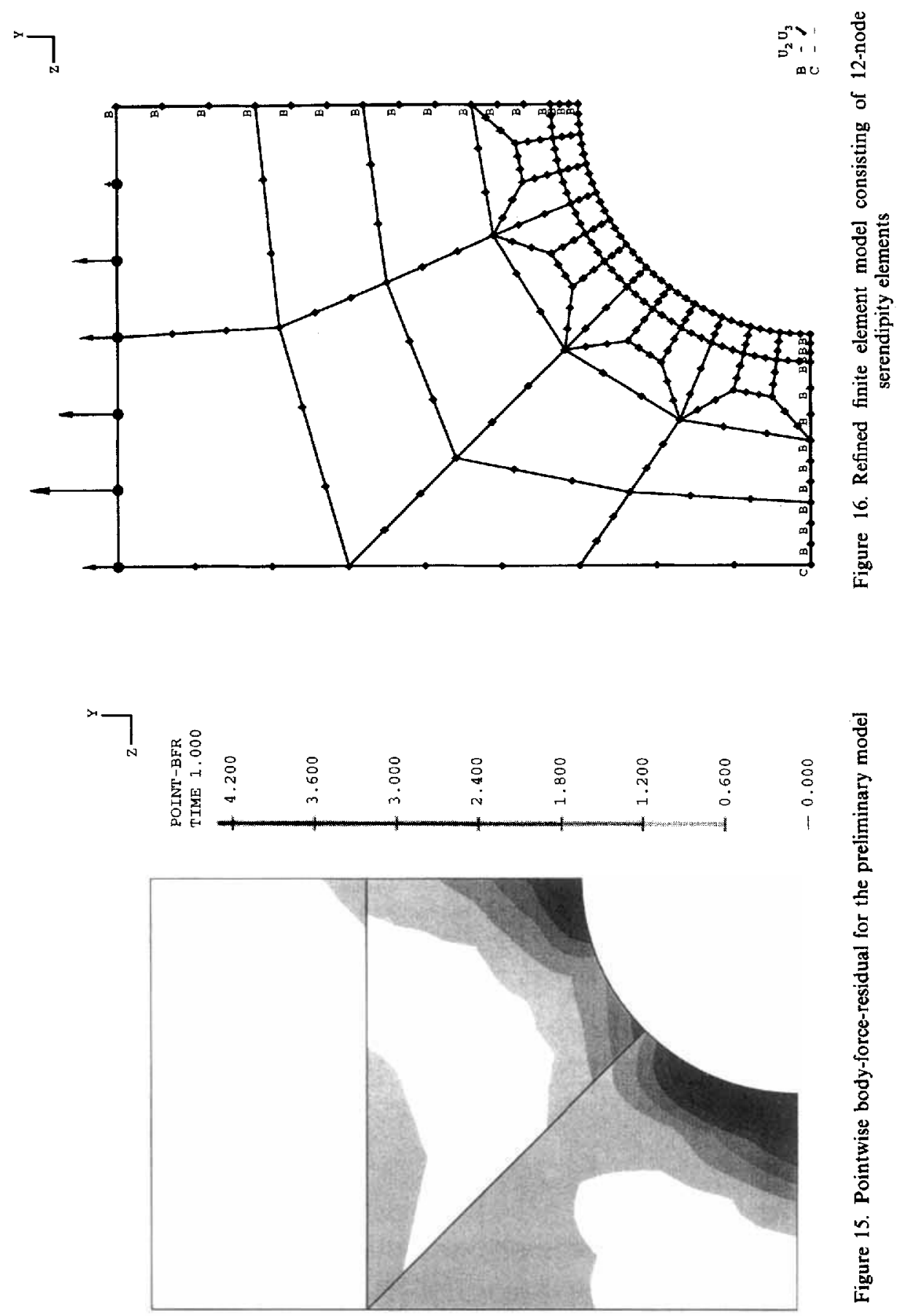

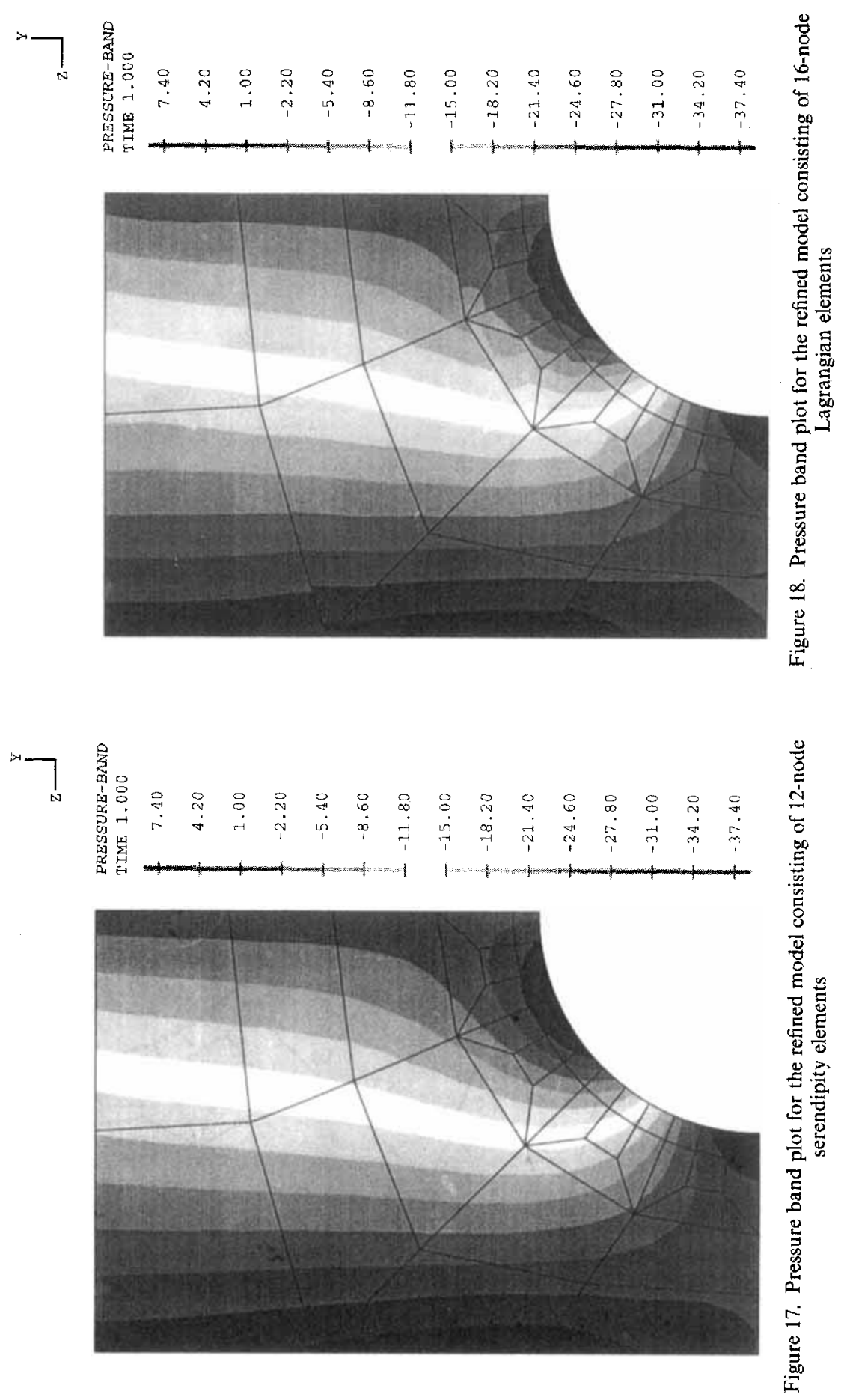
12-node serendipity elements are then used for the uniform p-refinement. The refined model is shown in Figure 16. The resulting pressure band plot is shown in Figure 17. By looking at the roughness of the bands, we can tell that the accuracy of the stress distribution is not high enough. From our previous discussion, we know that this can happen: the element distortions needed for the construction of the nearly optimal mesh have degraded the performance of the 12-node elements.

By simply replacing the 12-node elements with 16-node Lagrangian elements, we obtain the result shown in Figure 18. From the smoothness of the stress band plot, we can tell that we have arrived at the desired solution.

From this numerical example, we see that the insensitivity of the Lagrangian element to angular distortions has given it the 'flexibility' to adapt reliably to modelling requirements.

\section{PRACTICAL IMPLICATIONS AND CONCLUSIONS}

We have shown that the predictive capabilities of the Lagrangian isoparametric elements are not affected by angular distortions. The serendipity isoparametric elements, on the other hand, are very sensitive to such distortions. We have also shown that both types of elements are affected by curved-edge distortions. These findings have several important practical implications, such as:

1. For a reliable analysis, the use of the serendipity elements is best restricted to cases where rectangular or parallelogram elements can be used.

2. Lagrangian elements can be freely used: one need not be concerned about awkward-looking meshes as long as the elements have straight sides and evenly spaced nodes. This finding is of much value when considering mesh generation schemes.

3. Elements with curved sides should be used to represent only curved boundaries since the performance of such elements is inferior to those that have straight sides.

4. Lagrangian elements are preferred in large-displacement, large-strain analyses since the elements in these analyses can quickly become distorted.

5. Adaptive schemes in large deformation analysis should be based on the use of completely new meshes when mesh/interpolation refinement at a particular step is required. ${ }^{17}$ Straightsided Lagrangian elements and evenly spaced nodes within each element in the deformed configuration should be used since this can improve the accuracy of the solution.

In this paper, we have shown that, comparing the Lagrangian and the serendipity types of displacement-based isoparametric elements, the Lagrangian type is the more reliable and effective one for general use. This conclusion also means that $p$-type formulations should be based on Lagrangian interpolations.

Finally, we point out that this conclusion is fortuitous when considering almost incompressible analysis in that, for such analysis, the Lagrangian $u / p$ elements are also the more effective ones to use because of their stability and convergence properties. ${ }^{18,19}$

\section{REFERENCES}

1. J. A. Stricklin, W. S. Ho, E. Q. Richardson and W. E. Haisler, 'On isoparametric vs linear strain triangular elements', Int. j. numer, methods eng., 11, 1041-1043 (1977).

2. J. Bäcklund, 'On isoparametric elements', Int. j. numer. methods eng., 12, 731-732 (1978).

3. L. N. Gifford, 'More on distorted isoparametric elements', Int. j. numer. methods eng., 14, 290-291 (1979).

4. D. J. Burrows, 'A finite element shape sensitivity study', in K. J. Bathe and D. R. J. Owen (eds.), Reliability of Methods for Engineering Analysis, Pineridge Press, Swansea, 1986, pp. 439-456.

5. E. L. Wachspress, 'High-order curved finite elements', Int. j. numer. methods eng., 17, 735-745 (1981). 
6. E. L. Wachspress, 'Basic functions and evaluation of element characteristics', in A. K. Noor and W. D. Pilkey (eds.), State-of-the-art Surveys on Finite Element Technology, ASME, New York, 1983, pp. 25-56.

7. R. L. Taylor, J. C. Simo, O. C. Zienkiewicz and A. C. H. Chan, 'The patch test-a condition for assessing FEM convergence', Int. j. numer. methods eng., 22, 39-62 (1986).

8. J. Robinson, 'The CRE method of element testing and the Jacobian shape parameters', in K. J. Bathe and D. R. J. Owen (eds.), Reliability of Methods for Engineering Analysis, Pineridge Press, Swansea, 1986, pp. 407-424.

9. J. Robinson, 'Some new distortion measures for quadrilaterals', Finite Elements in Analysis and Design, 3, 183-197 (1987).

10. J. Robinson, 'Distortion measures for quadrilaterals with curved boundaries', Finite Elements in Analysis and Design, 4, 115-131 (1988).

11. J. Barlow, 'More on optimal stress points-reduced integration, element distortions and error estimation', Int. j. numer. methods eng., 28, 1487-1504 (1989).

12. O. C. Zienkiewicz and R. L. Taylor, The Finite Element Method, volume 1. McGraw-Hill Book Company, fourth edition, 1989.

13. T. Sussman and K. J. Bathe, 'Studies of finite element procedures-stress band plots and the evaluation of finite element meshes', Engineering Computations, 3, 178-191, 1986,

14. K. J. Bathe, Finite Element Procedures in Engineering Analysis, Prentice-Hall, Englewood Cliffs, N.J., 1982.

15. I. Babuška, B. A. Szabó and I. N. Katz, 'The p-version of the finite element method', SIAM J. Numer. Anal., 18, 515-545 (1981).

16. O. C. Zienkiewicz, J. Z. Zhu and N. G. Gong, 'Effective and practical $h$-p-version adaptive analysis procedures for the finite element method', Int. j. numer, methods eng., 28, 879-891 (1989).

17. K. Kato, N. S. Lee and K. J. Bathe, Adaptive finite element analysis of large strain elastic response, Comp. Struct., 47, 829-855 (1993).

18. T. Sussman and K. J. Bathe, 'A finite element formulation for nonlinear incompressible elastic and inelastic analysis', Comput. Struct., 26, 357-409 (1987).

19. F. Brezzi and K. J. Bathe, 'A discourse on the stability conditions for mixed finite element formulations', Comput. Methods Appl. Mech. Eng., 82 (1-3), 27-57 (1990). 